\title{
SiFBA5, a cold-responsive factor from Saussurea involucrata promotes cold resilience and biomass increase in transgenic tomato plants under cold stress
}

\author{
Jianqiang Mu ${ }^{1,2+}$, Yajuan Fư ${ }^{2 \dagger}$, Bucang Liu ${ }^{1}$, Yao Zhang ${ }^{1}$, Aiying Wang ${ }^{1}$, Yuxia Li ${ }^{1}$ and Jianbo Zhu ${ }^{1 *}$
}

\begin{abstract}
Background: Saussurea involucrata survives in extreme arctic conditions and is very cold-resistant. This species grows in rocky, mountainous areas with elevations of 2400-4100 m, which are snow-covered year-round and are subject to freezing temperatures. S. involucrata's ability to survive in an extreme low-temperature environment suggests that it has particularly high photosynthetic efficiency, providing a magnificent model, and rich gene pool, for the analysis of plant cold stress response. Fructose-1, 6-bisphosphate aldolase (FBA) is a key enzyme in the photosynthesis process and also mediates the conversion of fructose 1, 6-bisphosphate (FBP) into dihydroxyacetone phosphate (DHAP) and glycerol triphosphate (GAP) during glycolysis and gluconeogenesis. The molecular mechanisms underlying S. involucrata's cold tolerance are still unclear; therefore, our work aims to investigate the role of FBA in plant cold-stress response.
\end{abstract}

Results: In this study, we identified a cold-responsive gene, SiFBA5, based on a preliminary low-temperature, genome-wide transcriptional profiling of $S$. involucrata. Expression analysis indicated that cold temperatures rapidly induced transcriptional expression of SiFBA5, suggesting that SiFBA5 participates in the initial stress response. Subcellular localization analysis revealed that SiFBA5 is localized to the chloroplast. Transgenic tomato plants that overexpressed SiFBA5 were generated using a CaMV 355 promoter. Phenotypic observation suggested that the transgenic plants displayed increased cold tolerance and photosynthetic efficiency in comparison with wild-type plants.

Conclusion: Cold stress has a detrimental impact on crop yield. Our results demonstrated that SiFBA5 positively regulates plant response to cold stress, which is of great significance for increasing crop yield under cold stress conditions.

Keywords: SiFBA5, Calvin cycle, Photosynthesis, Cold stress, Chlorophyll

\footnotetext{
*Correspondence: zhujianboSHZU@163.com

${ }^{\dagger}$ Jianqiang Mu and Yajuan Fu contributed equally to this work.

${ }^{1}$ Key Laboratory of Agricultural Biotechnology, College of Life Science,

Shihezi University, Shihezi, Xinjiang 832003, China

Full list of author information is available at the end of the article
}

\section{$\triangle B M C$}

C C The Author(s). 2021 Open Access This article is licensed under a Creative Commons Attribution 4.0 International License, which permits use, sharing, adaptation, distribution and reproduction in any medium or format, as long as you give appropriate credit to the original author(s) and the source, provide a link to the Creative Commons licence, and indicate if changes were made. The images or other third party material in this article are included in the article's Creative Commons licence, unless indicated otherwise in a credit line to the material. If material is not included in the article's Creative Commons licence and your intended use is not permitted by statutory regulation or exceeds the permitted use, you will need to obtain permission directly from the copyright holder. To view a copy of this licence, visit http://creativecommons.org/licenses/by/4.0/ The Creative Commons Public Domain Dedication waiver (http://creativecommons.org/publicdomain/zero/1.0/) applies to the data made available in this article, unless otherwise stated in a credit line to the data. 


\section{Background}

The Calvin cycle is a critical part of photosynthetic carbon fixation, providing essential compounds for plant survival $[1,2]$. This process is associated with a variety of catalytic reactions and divided into three distinct phases: carbon fixation, reduction, and regeneration of the starting molecule [3]. It has been reported that plants grown in moderate light and temperature conditions can withstand more than a $50 \%$ reduction in ribulose-1, 5-biphosphate carboxylase-oxygenase (Rubisco) activity with little effect on photosynthesis. In contrast, under high light conditions, decreased Rubisco activity was related to the reduction of photosynthesis [4]. Rubisco is not always the most active enzyme during the Calvin cycle, other enzymes probably exert greater control over carbon flow during photosynthesis, specifically, sedoheptulose-1, 7-bisphosphatase (SBPase), transketolase (TK), and fructose-1,6-bisphosphate aldolase (FBA). Therefore, these enzymes are candidates for study in efforts to increase photosynthetic carbon fixation $[3,5]$.

FBA is a key metabolic enzyme that mediates the reversible conversion of fructose-1,6-bisphosphate (FBP) into dihydroxyacetone phosphate (DHAP) and glyceraldehyde-3-phosphate (GAP) during glycolysis and gluconeogenesis [6, 7]. In the Calvin cycle, FBA can also catalyze the condensation of sedoheptulose-1,7-bisphosphate from erythrose-4-phosphate and GAP [8, 9]. Antisense inhibition and photosynthesis modeling studies have shown that FBA may play a major role in controlling carbon metabolism rates and ribulose-1,5biphosphate (RuBP) regeneration in the Calvin cycle [10-12]. Experiments in tobacco (Nicotiana tabacum) indicated that overexpression of $F B A$ can promote RuBP regeneration while slightly increasing growth and biomass productivity [5]. Additionally, a study on tomato (Solanum lycopersicum) showed that SlFBA7 overexpression increased the activity of several major enzymes in the Calvin cycle, as well as the net photosynthetic rate (Pn), seed size, and stem diameter [13].

FBA is also involved in plant cold-stress response. For instance, cold stress upregulates $F B A$ family member ClAldC expression in Codonopsis lanceolata [14]. Interestingly, the $F B A$ family responds differently to cold treatment in Arabidopsis and rice (Oryza sativa) [13, 15]. Despite extensive research on several model plants and commercial crops, few studies have explored the role of $F B A$ in more extreme habitats.

$S$. involucrata is a perennial alpine plant resistant to extreme cold stress [16]. Despite having a short life cycle, S. involucrata can accumulate substantial biomass and has evolved numerous genes specifically associated with cold tolerance [17]. Recently, transcriptome sequencing and extensive bioinformatics analysis were completed for S. involucrata, providing us with an opportunity to determine the specific $F B A$ gene associated with S. involucrata cold stress tolerance [18]. In the present study, we used Agrobacterium-mediated transformation to construct transgenic lines of tomato plants to study the putative function of SiFBA5 genes [19, 20].

\section{Results}

\section{Isolation of siFBA5 from S. involucrata}

SiFBA5 cDNA was identified from transcriptome sequencing of S. involucrata. Full-length cDNA was obtained using gene-specific primers [17]. Sequencing analysis revealed that the open reading frame (ORF) of SiFBA5 cDNA (1179 bp) encodes a 392-amino-acid protein, with a molecular mass of $42.57 \mathrm{kDa}$ and isoelectric point (pI) of 8.84 (Figure S1-S2). Multiple sequence alignment revealed that SiFBA5 shared 75.2\%, 74.9\%, and $70.2 \%$ similarity to Compositae homologs $C c F B A 3$, $\angle S F B A 3$, and $H a F B A 3$, respectively; thus, the gene was highly conserved (Fig. 1a). The gene also exhibited the canonical lysine residue of Class I aldolases involved in aldol condensation. Phylogenetic analysis indicated that SiFBA5 is closely related to the homolog AtFBA3, a chloroplastic FBA (Fig. 1b). This outcome suggests that SiFBA5 localizes to the chloroplast.

\section{Expression patterns of SiFBA5}

To investigate how SiFBA5 responds to cold stress, $S$. involucrata rosettes at the five-leaf stage were exposed to $4{ }^{\circ} \mathrm{C}$ for $1,3,6,12$, and $24 \mathrm{~h}$. After $1 \mathrm{~h}$, the relative expression of SiFBA5 increased 10-fold, before gradually declining (Fig. 2a). This rapid and strong induction of expression suggested that SiFBA5 participates in the initial response to cold stress.

\section{Subcellular localization of SiFBA5}

WoLF PSORT (https://wolfpsort.hgc.jp/), YLoc (www. multiloc.org/YLoc), and MultiLoc2 (http://www-bs. informatik.uni-tuebingen.de/Services/MultiLoc2) predicted that SiFBA5 subcellular localization was in the chloroplast. To test the robustness of this prediction, we constructed a SiFBA5-GFP fusion protein and used confocal microscopy to assay its presence in Nicotiana benthamiana leaf cells. We confirmed that SiFBA5-GFP was localized to the chloroplast (Fig. 2b). Therefore, SiFBA5 likely participates in the Calvin cycle and may be related to downstream gene regulation during photosynthetic carbon flux.

\section{Cold stress responses of transgenic SiFBA5 overexpression lines}

To reveal the functional roles of SiFBA5, we constructed tomato overexpression lines under the control of the $35 \mathrm{~S}$ promoter. Analysis with PCR verified transgene integration. Under normal temperature conditions $\left(25^{\circ} \mathrm{C}\right)$, 

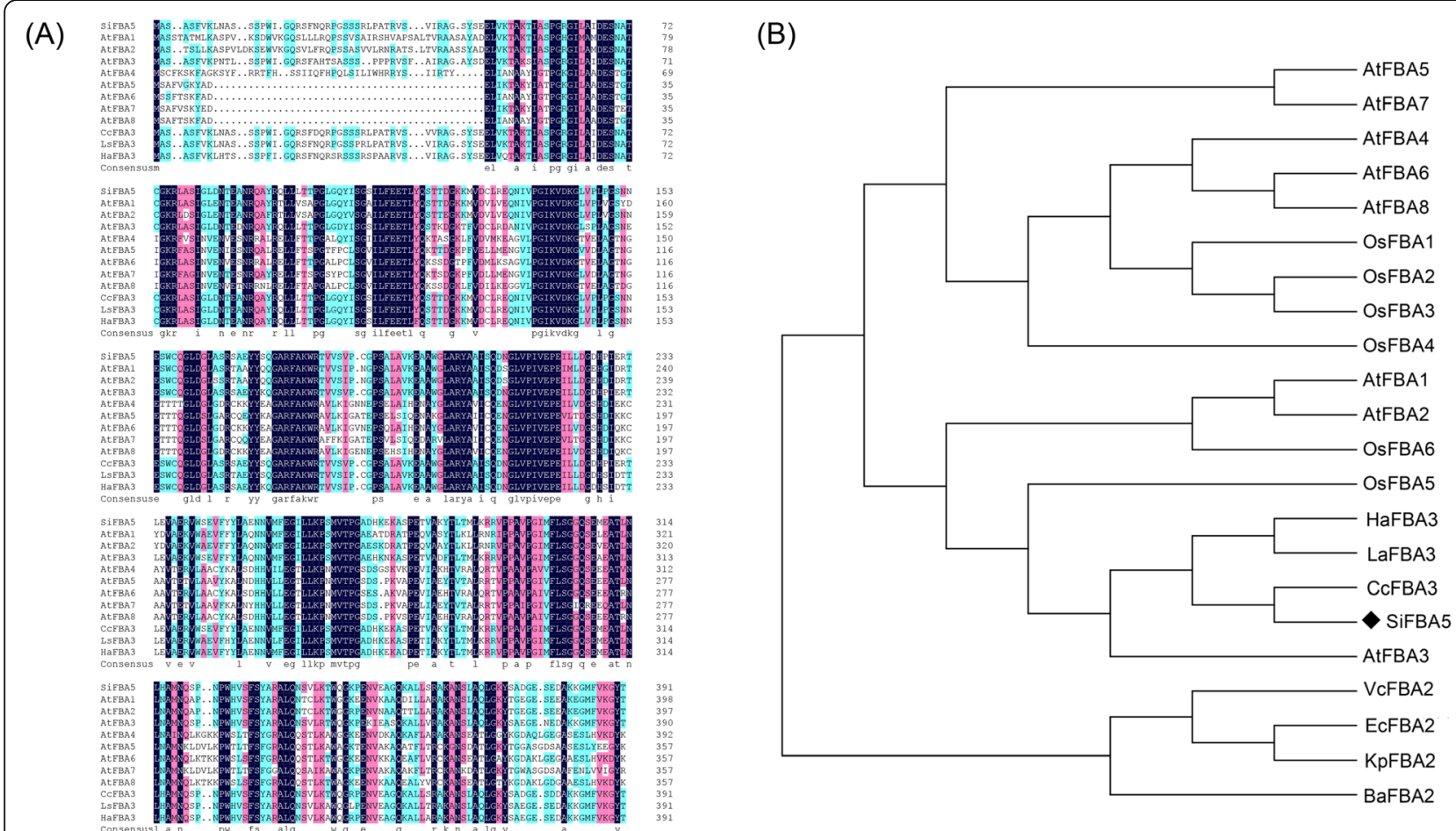

Fig. 1 Sequence alignment and phylogeny of SiFBA5 homologs. a Amino acid alignment of SiFBA5 homologs. b Phylogenetic assay of FBA homologous proteins across species. The following species were included: Arabidopsis thaliana, Oryza sativa, Cynara cardunculus, Lactuca sativa, Helianthus annuus, Escherichia coli, Klebsiella pneumonia, Brucella abortus, and Vibrio celticus

growth did not differ between overexpression lines and wild type, suggesting that excess SiFBA5 does not cause an aberrant phenotype (Fig. 3). However, during cold tolerance assays $\left(4^{\circ} \mathrm{C}\right)$, wild-type plants exhibited thinning and drooping leaves, along with bent side-branches, whereas overexpressing SiFBA5 plants did not. Furthermore, at $0{ }^{\circ} \mathrm{C}$, wild-type leaves curled and deepened in color, while transgenic leaves were no longer viable (Fig. 3).

\section{Physiological changes in transgenic plants under cold stress}

Cold stress causes the overproduction of reactive oxygen species (ROS) and oxidative damage in plant cells. Under
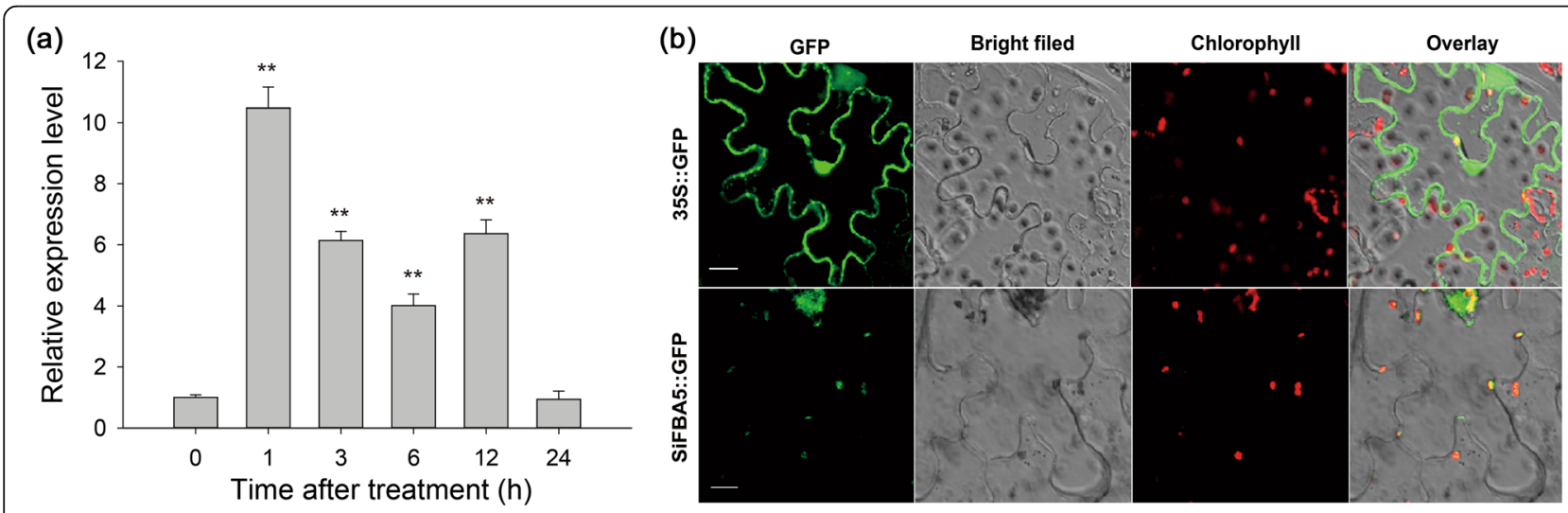

Fig. 2 Expression patterns of SiFBA5 under cold stress and its subcellular localization. a qRT-PCR analysis of SiFBA5 in S. involucrata. Means \pm SD was obtained from three biological replicates. ${ }^{*}, P<0.05 ;{ }^{* *}, P<0.01$. b Subcellular localization of SiFBA5 in tobacco epidermal cells. SiFBA5-GFP and empty control vector (35S::GFP) were transiently expressed in tobacco epidermal cells. Fluorescent images were taken by confocal microscopy after Agrobacterium-mediated infiltration for $48 \mathrm{~h}$. Upper row, the control panel of 355::GFP, lower row, 355::-SiFBA5-GFP. From left to right: channels of GFP fluorescence, bright field, chloroplast autofluorescence and an overlay of all three. Scale bar $=20 \mu \mathrm{m}$. One-way ANOVA was used to compare the statistical difference between measurements $(P<0.05)$ 


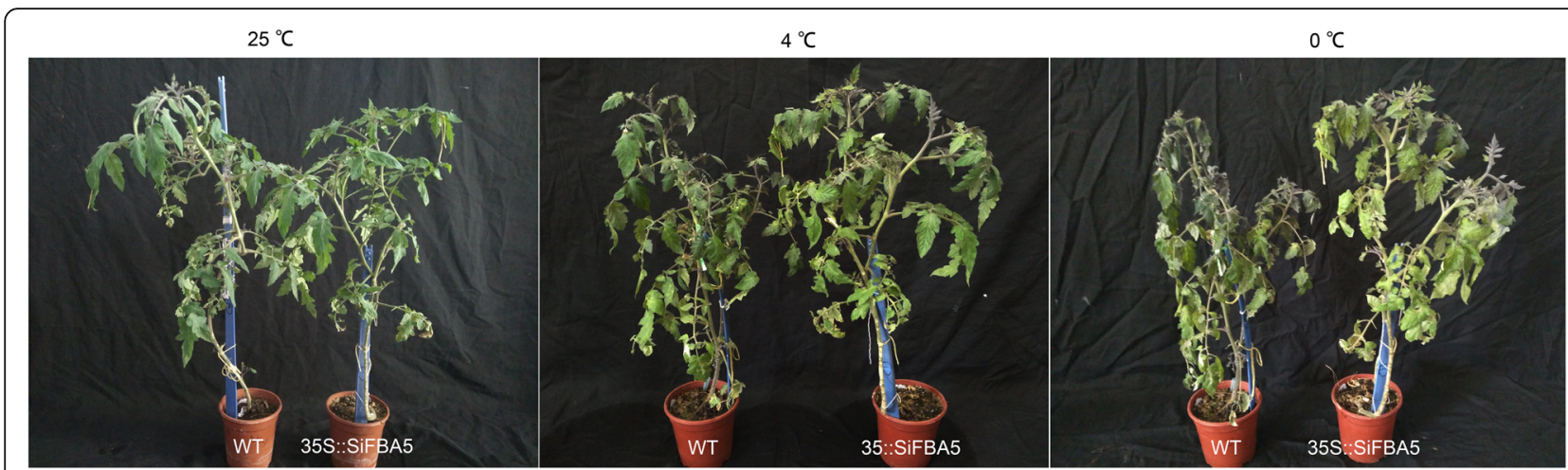

Fig. 3 Stress responses of SiFBA5 transgenic plants under cold. Nine-week-old WT and SiFBA5 transgenic plants were subjected to either normal temperatures $\left(25^{\circ} \mathrm{C}\right)$ or cold stress conditions $\left(4^{\circ} \mathrm{C}\right.$ and $\left.0^{\circ} \mathrm{C}\right)$ for $24 \mathrm{~h}$ before taking pictures

cold stress conditions $\left(4^{\circ} \mathrm{C}\right)$, the overexpression lines exhibited significantly less malondialdehyde (MDA) accumulation than wild type $(P<0.05)$ (Fig. 4a). Since MDA is the product of ROS-induced lipid peroxidation, this outcome indicated that the plants were subject to less oxidative stress when SiFBA5 was overexpressed. We then assayed the activities of three major antioxidant enzymes (superoxide dismutase, SOD; catalase, CAT; and peroxidase, $\mathrm{POD}$ ) to investigate the cause of this change. There was no significant difference in SOD activity between transgenic and wild-type plants under normal conditions, but under cold stress, SOD activity was significantly enhanced $(P<0.05)$ (Fig. $4 \mathrm{~b})$. Additionally, we found that POD activity increased significantly in the transgenic lines compared with the wild type under cold stress $(P<0.05)($ Fig. $4 \mathrm{c})$. As temperatures decreased, the corresponding decrease in CAT activity was more pronounced than the decreases in SOD and POD activity. Transgenic and wild type plants again differed significantly, with less CAT activity in the former than in the latter $(P<0.05) \quad$ (Fig. 4d). Our results suggest that SiFBA5 overexpression inhibits ROS damage under cold stress by both decreasing MDA accumulation and enhancing antioxidant enzyme activity.

\section{Effects of SiFBA5 on photosynthetic and chlorophyll fluorescence factors}

We determined changes in net photosynthetic rate $(\mathrm{Pn})$ to delve deeper into how cold stress alters photosynthetic efficiency. Our findings showed that transgenic plants and wild-type plants did not differ significantly in Pn under normal conditions. However, at $10{ }^{\circ} \mathrm{C}$ and $4{ }^{\circ} \mathrm{C}$, overexpression lines displayed significantly higher $\mathrm{Pn}$ than wild type $(P<0.01)$ (Fig. $4 \mathrm{~g})$. In line with this observation of elevated photosynthesis under SiFBA5 overexpression, shoot and root dry biomass were respectively 84.34 and $40.14 \%$ greater in transgenic plants than in wild type $(P<0.01)$ (Fig. 4 h). We also observed significantly higher total chlorophyll content in overexpression lines than in wild type at $16^{\circ} \mathrm{C}$ and $10^{\circ} \mathrm{C}(P<$ $0.05)$ (Fig. 4e). Finally, we measured the maximum efficiency of PSII photochemistry $(\mathrm{Fv} / \mathrm{Fm})$, which reflects PSII photoinhibition. In the SiFBA5 overexpression lines, $\mathrm{Fv} / \mathrm{Fm}$ was greater than in wild-type plants $(P<0.05)$ (Fig. 4f), indicating that cold stress did not damage the PSII complex in transgenic plants.

\section{SiFBA5 overexpression effects on other Calvin-cycle enzymes}

We analyzed the expression of several enzymes involved in photosynthesis regulation: Rubisco, SBPase, fructose-1, 6-bisphosphatase (FBPase), glyceraldehyde-3-phosphate dehydrogenase (GAPDH), and triose-3-phosphate isomerase (TPI). Transgenic lines showed significantly higher expression of both $r b c L$ and $r b c S$ (Rubisco large and small subunits) than wild type at $4{ }^{\circ} \mathrm{C}(P<0.05)$ (Fig. 5a, b). Additionally, SBPase, FBPase, GAPDH, and TPI expression was significantly higher in transgenic lines than in wild type $(P<0.05)$ (Fig. 5c-f).

\section{Discussion}

Plants are sessile and cannot avoid damage in their environment by moving, so they have evolved a series of genes to respond to stress. A previous study found that the use of Agrobacterium-mediated transformation of Persian walnut (Juglans regia) somatic embryos can enhance the resistance response of the plants and contribute to better appearance of the kernels for consumers [21-23]. Freezing injury is a major cause of crop loss and a limiting factor. There are some studies regarding cold hardiness mechanisms and methods of cold adaptation in walnut [24-26].

Accordingly, this study is designed to exploit the $F B A$ gene from $S$. involucrata and transfer it into tomato plants. Fructose-1,6-bisphosphate aldolase is critical to carbon metabolism in plants, playing a role in processes 
(a)

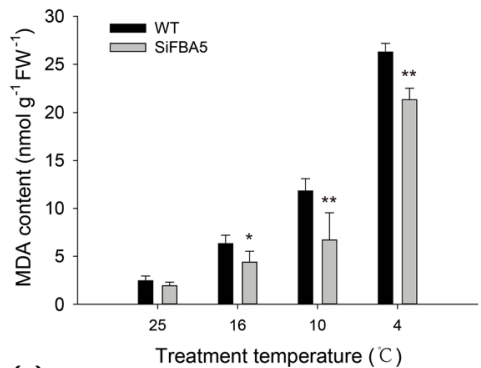

(c)

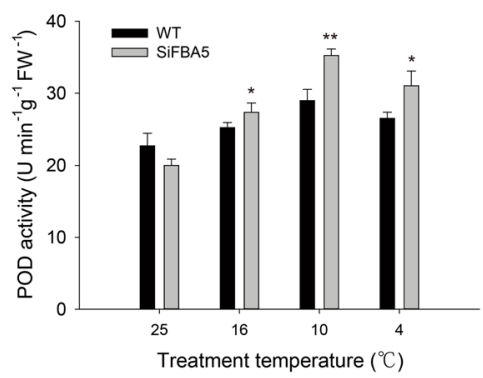

${ }^{(e)}{ }^{4}$

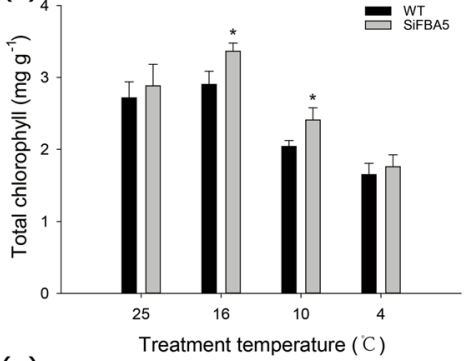

(g)

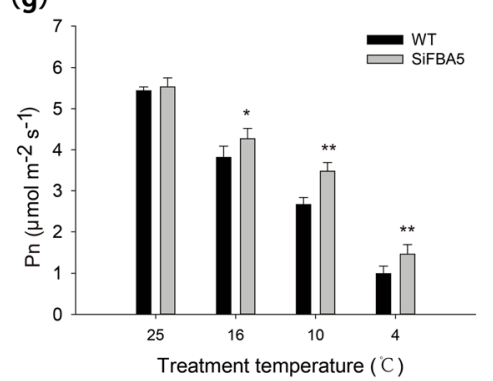

(b)

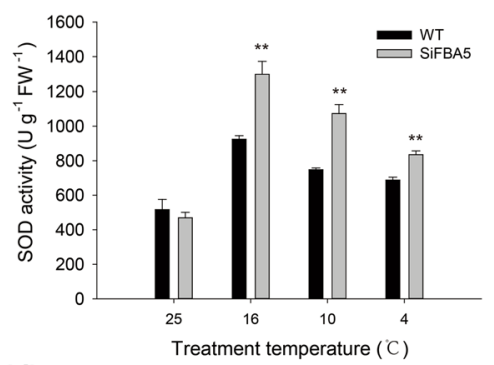

(d)

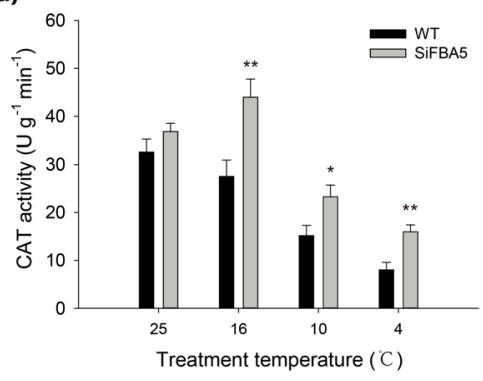

(f)

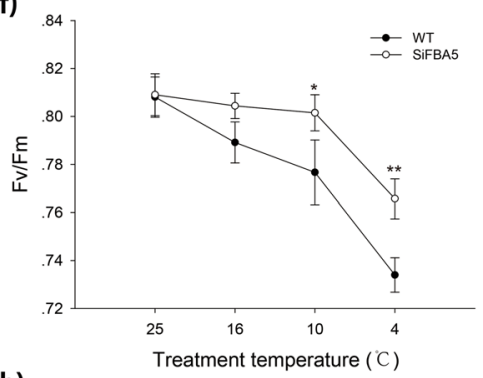

(h)

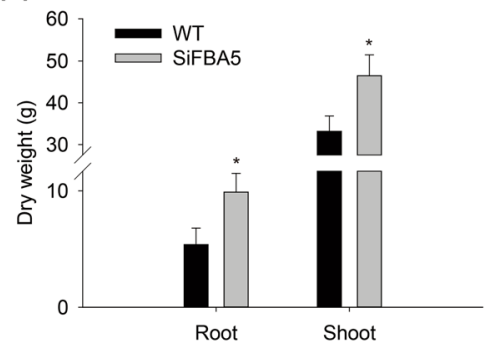

Fig. 4 Determinations of various physiological parameters and biomass production in WT and transgenic tomato plants. a-e Physiological analysis of WT and SiFBA5 transgenic plants under normal temperature $\left(25^{\circ} \mathrm{C}\right)$ and cold stress $\left(16,10\right.$, and $\left.4{ }^{\circ} \mathrm{C}\right)$, a malondialdehyde (MDA), b superoxide dismutase (SOD), c peroxidase (POD), $\mathbf{d}$ catalase (CAT), and e total chlorophyll content. $\mathbf{f}$ Maximal efficiency of PSIl photochemistry (Fv/Fm), $\mathbf{g}$ net photosynthetic rate $(\mathrm{Pn}), \mathbf{h}$ biomass. Mean \pm SD was obtained in one experiment with three biological replicates. Asterisks indicate significant differences between WT and transgenic plants $\left({ }^{*} P<0.05 ;{ }^{* *} P<0.01\right)$. One-way ANOVA was used to compare the statistical difference between measurements $(P<0.05)$

such as glycolysis, gluconeogenesis, pentose phosphate pathway, and the Calvin cycle [7]. Studies in multiple plant species have amply demonstrated the critical role of $F B A$ in regulating plant stress-tolerance, growth, and development $[5,14,27,28]$. To the best of our knowledge, the present study is one of the first studies to examine $F B A$ function in a plant species endemic to an extreme habitat, specifically $S$. involucrata, which can survive even at temperatures below $-21^{\circ} \mathrm{C}$ [29]. Transcriptome analysis of $S$. involucrata identified 15 FBA gene transcripts, three of which were upregulated in response to cold stress (data not shown). We selected SiFBA5 here for further analysis. A bioinformatics analysis revealed that $S i F B A 5$ proteins are highly similar to FBA homologs in other species. Consistent with the low-temperature transcriptome profile, cold stress 

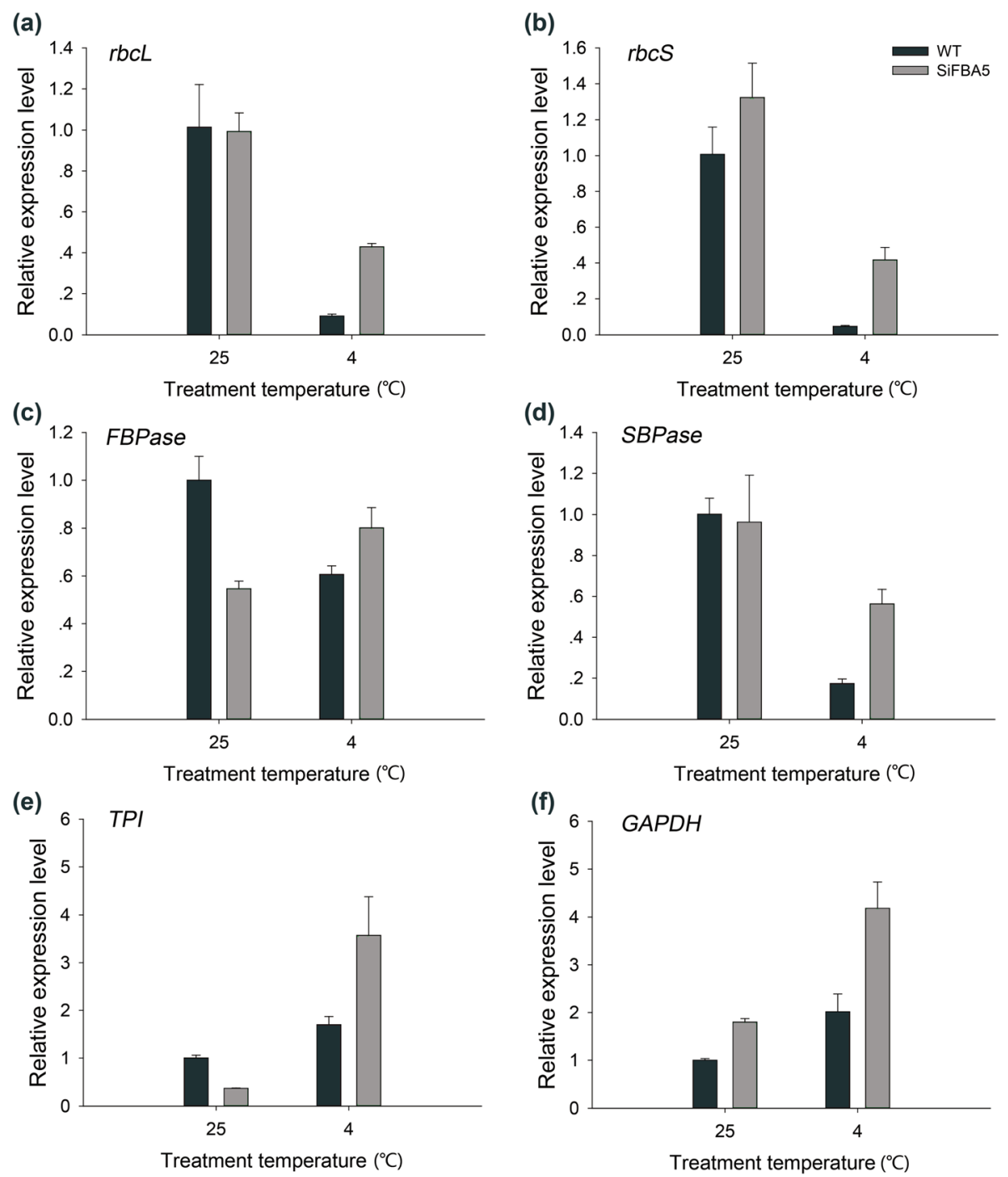

Fig. 5 Transcriptional expression analyses of primary Calvin-cycle enzyme encoding genes in WT and transgenic tomato plants. a rbcL (Gene ID:101265242), b rbcS (Gene ID:543973), c FBPase (Gene ID:101264273), d SBPase (Gene ID:100316873), e TPI (Gene ID:778306), and f GAPDH (Gene ID:100736499). Values are mean \pm SD $(n=3)$. One-way ANOVA was used to compare the statistical difference between measurements $(P<0.05)$

significantly increased SiFBA5 transcript abundance. Phylogenetic analysis of SiFBA5 revealed highly homologous relationships with chloroplast FBAs in plants such as Arabidopsis, rice, tobacco, and tomato [5, 13, 15]. In higher plants, FBAs have two isozymes: cytosolic and chloroplast [15], the latter is involved in the Calvin cycle. In potatoes, suppression of chloroplast FBA inhibits photosynthesis [30]. Additionally, the SiFBA5-GFP fusion protein was localized to the chloroplast (Fig. 2b). Our SiFBA5-overexpression transgenic lines revealed improved photosynthetic efficiency and cold tolerance compared with wild-type plants under low-temperature exposure.

We also successfully identified some of the physiological processes involved in the cold stress response, such as increased MDA production. Therefore, we tested the MDA content in wild type and SiFBA5overexpression transgenic lines under cold stress, which revealed a decrease in the MDA content of SiFBA5-overexpression transgenic lines relative to wild type $(P<0.05)$. These results indicated that SiFBA5-overexpressing plants had lower oxidative stress (Fig. 4a) [31]. Furthermore, under cold stress, transgenic plants had significantly higher SOD, POD, and CAT activity than in the wild type. These three enzymes are part of the antioxidant defense system that evolved in plants to counteract ROS-induced oxidative damage (e.g., lipid peroxidation) [32]. Given that abiotic stress increases the activity of these enzymes; we speculated that overexpression of SiFBA5 
can eliminate excess ROS from cells, offering protection against oxidative damage.

Low temperatures decrease plant capacity to use light energy and increase photoinhibition in cold-sensitive plants [33]. Some data suggest that photosynthesis is inhibited through reduced aldolase expression [10]. Another study found that $A t F B A$ overexpression specifically promoted $\mathrm{RuBP}$ regeneration, $\mathrm{CO}_{2}$ fixation efficiency, and photosynthetic rate in transgenic tobacco [5]. Our results demonstrated that SiFBA5 overexpression lines under cold stress constitutively elevated $F B A$ expression in chloroplasts and promoted Rubisco expression, which enhanced RuBP regeneration, leading to accelerated RuBP metabolism. These changes then promote the entire photosynthetic pathway. Variation in the individual FBA of transgenic plants showed that the regenerative capacity of the C3 cycle places limitations on photosynthetic rate [3].

Cold stress decreases $\mathrm{Fv} / \mathrm{Fm}$, making the latter an important variable in screening for cold tolerance [34]. Our study confirmed that cold stress reduces $\mathrm{Fv} / \mathrm{Fm}$, resulting in plants that cannot efficiently use excitation energy and thus exhibit reduced PSII photochemical efficiency. However, transgenic plants had higher Fv/Fm than wild type under cold stress. The ability to sustain proportionately higher $\mathrm{Fv} / \mathrm{Fm}$ could enhance photosynthesis after a cold-stress-induced slowdown. We also found that overexpression of chloroplast FBA slightly increased Pn under cold stress, but not under normal temperatures. Thus, prolonged exposure to cold stress appeared to reduce SiFBA5 transcription, but the overexpression of SiFBA5 partially compensated for FBA loss, which is in line with data from potatoes [30] and tomatoes [35].

\section{Conclusion}

We successfully cloned SiFBA5 from S. involucrata and identified the gene product as an aldolase. Overexpressing SiFBA5 in tomato alleviated cold-stress-induced cellular damage, improved cold tolerance, and improved photosynthetic capacity under cold stress conditions. Our data provide a theoretical foundation and experimental basis for using SiFBA5 in the development of $S$. involucrata germplasm resources. Moreover, the gene is a strong candidate for genetic engineering to increase crop production and stress resistance in cold climates.

\section{Methods}

\section{Plant materials and treatments}

S. involucrate seeds were a gift from Zhaosu County Forestry Bureau, Yili Prefecture, Xinjiang. Seedlings of $S$. involucrata were cultured using plant tissue culture technology and placed in a glasshouse at $19^{\circ} \mathrm{C}$ with a 16 $\mathrm{h}$ light $/ 8 \mathrm{~h}$ dark photoperiod.
Nicotiana benthamiana seeds were grown in pots with 3:1 peat moss and vermiculite soil $(\mathrm{v} / \mathrm{v})$ under a photoperiod of $12 \mathrm{~h}$ light at $26^{\circ} \mathrm{C} / 12 \mathrm{~h}$ dark at $22^{\circ} \mathrm{C}$, with $50 \%$ relative humidity.

Tomato (Yaxin 87-5) seeds were surface-sterilized in $75 \%$ ethanol for $1 \mathrm{~min}, 2 \% \mathrm{v} / \mathrm{v}$ sodium hypochlorite for $15 \mathrm{~min}$, and rinsed six times with sterile distilled water. Seeds were sown on 1/2 Murashige and Skoog (MS) medium. For a week, seeds were incubated at $25^{\circ} \mathrm{C}$ under a $16 \mathrm{~h}$ light $/ 8 \mathrm{~h}$ dark cycle with fluorescent lights. Cotyledon explants of 7-d-old seedlings were used for Agrobacterium transformation. For cold treatment, rosettes at the five-leaf stage were exposed to $4{ }^{\circ} \mathrm{C}$ for 1,3 , 6,12 , and $24 \mathrm{~h}$, respectively. All tissue samples were immediately frozen in liquid nitrogen and stored at $-80^{\circ} \mathrm{C}$.

\section{RNA isolation and qRT-PCR}

Total RNA was isolated with TRIzol from S. involucrate and tomato, following manufacturer protocol. PrimeScriptTM RT reagent Kit (TaKaRa, China) was used for first-strand cDNA synthesis. Quantitative real-time PCR (qRT-PCR) was performed in the Light Cycler 480 system (Roche Diagnostics, Germany) using LightCycler 480 SYBR Green I master mix (Roche Diagnostics). The thermocycling protocol was 40 cycles of $95^{\circ} \mathrm{C}$ for $10 \mathrm{~s}, 60^{\circ} \mathrm{C}$ for $20 \mathrm{~s}$, and $72{ }^{\circ} \mathrm{C}$ for $30 \mathrm{~s}$. Relative expression was calculated with the $2^{-\Delta \Delta C t}$ method. Each expression profile was independently verified in triplicate. qRT-PCR primers are shown in Supplementary Table S1. We used S. involucrat cDNA to amplify the $F B A$ gene and cDNA from tomato to detect the expression of related genes. The experiment is represented as means \pm SE of three replications.

\section{Isolation of SiFBA5 gene from S. involucrata}

The SiFBA5 gene sequence was obtained from SaussureaKBase (http://www.shengtingbiology.com/ SaussureaKBase/index.jsp.). Putative full-length SiFBA5 cDNA and genomic DNA were amplified using genespecific primers SiFBA5-F and - $\mathrm{R}$ (Supplementary Table S1). SiFBA5 cDNA was ligated into the $\mathrm{pMD}^{\circ} 19-\mathrm{T}$ simple vector (TaKaRa, China) and E. coli DH5 $\alpha$ competent cells (TransGen, China); successful cloning was verified via Sanger sequencing (BGI, Beijing, China). All subsequent constructs were made using this clone as a template. SiFBA5 gene homologs were identified using BlastP in NCBI (https://blast.ncbi.nlm.nih.gov/Blast. cgi). Multiple sequence alignments were performed in ClustalX2 with default parameters and DNAMAN. Alignments were then adjusted for constructing the phylogenetic tree in MEGA 5.0. 


\section{DNA constructs}

The coding region of SiFBA5 was amplified using primers G-SiFBA5-F and -R, with appropriate restriction sequences (Table S1). PCR products were digested with $\mathrm{BamH}$ I and Sal I, then ligated with the multiple cloning site (MCS) of $p C A M B I A 2300$ binary vector, containing GFP as the reporter under the control of cauliflower mosaic virus 35S (CaMV 35S) promoter [36]. The construct for SiFBA5 subcellular location was generated through the in-frame fusion of complete SiFBA5 CDS (stop codon TAG deleted) with GFP. To generate the overexpression construct, SiFBA5 ORF was cloned into the $p C A M B I A 2300$ vector under the control of the $C a M V$ $35 \mathrm{~S}$ promoter. The insert was released from pMD19-TSiFBA5 through Sma I and Sal I digestion, and then ligated into $p C A M B I A 2300$ MCS. Constructs were introduced into Agrobacterium strain GV3101 through electroporation using the GENE PULSER II system (BioRad, Hercules, CA, USA).

\section{Plant transformation}

The 35S::SiFBA5 vectors were introduced into a tomato for overexpression lines, respectively. Select sterile, robust tomato seedlings with fully expanded cotyledons, cut off the two ends of the cotyledons, and then divide them into two from the middle of the leaves. Use the leaf discs as genetically transformed explants, and spread them on $\mathrm{MS}+2 \mathrm{mg} / \mathrm{L} 6-\mathrm{BA}+0.15 \mathrm{mg} / \mathrm{L}$ IAA medium with the backside down, dark culture for 2 days. Transformation into the tomato, with cotyledons as explants, followed published protocols [37]. Callus induction and shoot regeneration were induced. Next, inoculated explants were screened on MS medium supplemented with different concentrations of plant growth regulators and antibiotics. Shoots were regenerated on a selective medium containing kanamycin $\left(50 \mathrm{mg} \mathrm{L}^{-1}\right)$. Rooted kanamycin-resistant primary transformants $(5-10 \mathrm{~cm}$ high) were transferred to potting soil; SiFBA5 presence was confirmed via PCR. The 35S::SiFBA5-GFP vectors were introduced into tobacco for subcellular location experiments, the third to fifth youngest leaves of the three- to four-week-old Nicotiana benthamiana (approximately 8-10 leaf stage) was injected with Agrobacterium-expressing GFP-fusion protein using a $1 \mathrm{~mL}$ syringe. Following 2-3 d incubation in growth chambers, a laser scanning confocal microscope (Zeiss, Germany) was used to image GFP fluorescence in tobacco leaves.

\section{Stress treatment}

Stress treatments were initiated after plants were grown for 9 weeks in the soil at $25^{\circ} \mathrm{C}$ under a $16 \mathrm{~h} / 8 \mathrm{~h}$ (light/ dark) photoperiod. To investigate the role of SiFBA5 in cold tolerance, wild-type and transgenic tomato plants were incubated (Percival Scientific, USA) for a week under a $12 / 12 \mathrm{~h}$ light $\left(25^{\circ} \mathrm{C}\right) /$ dark $\left(22^{\circ} \mathrm{C}\right)$ cycle with moderate light intensity and $50-60 \%$ relative humidity. Then, wild-type and transgenic tomato plants were subjected to cold stress at 16,10 , and $4{ }^{\circ} \mathrm{C}$, each for $3 \mathrm{~d}$. Subsequently, plants were placed overnight in a chamber set to $0{ }^{\circ} \mathrm{C}$.

\section{Physiological measurements}

Physiological traits were measured at different stages after cold stress treatments. Analysis of stress-induced oxidative damage used an MDA assay as previously described [38]. The nitro blue tetrazolium (NBT) photoreduction method was used to estimate SOD activity [39]. Next, POD activity was determined through monitoring increases in absorbance at $470 \mathrm{~nm}$ during guaiacol oxidation [40]. Finally, CAT activity was assayed through monitoring decreases in $\mathrm{H}_{2} \mathrm{O}_{2}$ absorbance $(240 \mathrm{~nm})$ within $1 \mathrm{~min}$ [41]. Total chlorophyll contents were measured following a previous method with some modifications [33]. We weighed out $0.1 \mathrm{~g}$ of leaf tissue into pieces, total chlorophyll was extracted using ethanol: acetone: $\mathrm{H}_{2} \mathrm{O}$ (4.5:4.5:1) and analyzed by UV spectrophotometry.

\section{Gas-exchange and chlorophyll fluorescence}

Net photosynthesis and stomatal conductance were detected using the portable photosynthesis system LI-6400 (LI-COR, USA). Chlorophyll fluorescence was measured with a Mini PAM chlorophyll fluorometer (Waltz, Germany) [42].

\section{Statistical analysis}

All data are represented as means \pm SE of three replications. SPSS $_{13.0}$ software (SPSS, Chicago, USA) was used to compare MDA content, SOD activity, POD activity, CAT activity, or relative gene expression in the control and transgenic lines. One-way analysis of variance followed by Tukey's honestly significant difference $(P<$ 0.05 and $P<0.01)$ multiple comparison tests were used to determine the significant differences. Error bars in all figures represent standard deviations from the mean. SigmaPlot ${ }_{12.0}$ was used to generate figures.

\section{Supplementary Information}

The online version contains supplementary material available at https://doi. org/10.1186/s12870-021-02851-8.

Additional file 1: Figure S1. Nucleotide and deduced amino acid sequences of SiFBA5. The numbers of Nucleotide and amino acid are shown on the left

Additional file 2: Figure S2. PCR identification of transgenic tomato line. (a) PCR assay of the transgenic plants; (b) RT-PCR assay of the transgenic plants. M: DNA Marker; Number means different transgenic lines; "+" The positive plasmid; WT: wild-type tomato plant. 
Additional file 3: Table S1. List of primers used in this study.

\section{Abbreviations}

qRT-PCR: Quantitative Real-Time PCR; SBPase: Sedoheptulose-1,7Bisphosphatase; TK: Transketolase; FBA: Fructose-1,6-Bisphosphate Aldolase; FBP: Fructose-1,6-Bisphosphate; DHAP: Dihydroxyacetone Phosphate; GAP: Glyceraldehyde-3-Phosphate; RuBP: Ribulose-1,5-Biphosphate; Pn: Photosynthetic rate; MS: Murashige and Skoog; ROS: Reactive Oxygen Species; MDA: Malondialdehyde; SOD: Superoxide Dismutase; CAT: Catalase POD: Peroxidase; MCS: Multiple Cloning Site; NBT: Nitro Blue Tetrazolium

\section{Acknowledgments}

We are grateful to Jin Wang, director of Wulanwusu Agrometeorological Experiment Station of Xinjiang for providing us with the apparatus for the assay of chlorophyll fluorescence and photosynthesis.

\section{Authors' contributions}

JZ, JM and YF planned the experiments and contributed significantly to writing the manuscripts. JM, YF, BL and YZ conducted the experiments. AW and $\mathrm{YL}$ conducted a few of the experiments. All authors have read and approved the final manuscript.

\section{Funding}

This work was supported by the National Science Foundation Project (31360053) and the National major Transgenic Project of China (2019ZX08005-004-009). The funding bodies had no role in the design of the study and collection, analysis, and interpretation of data and in writing the manuscript.

\section{Availability of data and materials}

All data generated or analyzed during this study are included in this published article and its supplementary information files. Also, original images are available from the corresponding author upon reasonable request (Jianbo Zhu, zhujianboSHZU@163.com).

\section{Ethics approval and consent to participate}

Plant materials (Saussurea involucrate) used in this study were from our lab (Key Laboratory of Agricultural Biotechnology, College of Life Science, Shihezi University, Shihezi 832003, Xinjiang, China; Email zhujianboSHZU@163.com). All plant materials were provided free of charge. S. involucrate used in this experiment were from tissue culture plantlets in our laboratory, it is not an endangered materials or species.

\section{Consent for publication}

Not applicable.

\section{Competing interests}

The authors declare that they have no competing interests.

\section{Author details}

${ }^{1}$ Key Laboratory of Agricultural Biotechnology, College of Life Science, Shihezi University, Shihezi, Xinjiang 832003, China. ²Department of Biology, Southern University of Science and Technology, Shenzhen 518055, Guangdong, China.

\section{Received: 19 August 2020 Accepted: 24 January 2021}

\section{Published online: 04 February 2021}

\section{References}

1. Geiger DR, Servaites JC. Diurnal regulation of photosynthetic carbon metabolism in C3 plants. Annu Rev Plant Biol. 1994;45:235-56.

2. Sharkey TD. Discovery of the canonical Calvin-Benson cycle. Photosynth Res. 2019:140:235-52.

3. Raines CA. The Calvin cycle revisited. Photosynth Res. 2003;75:1-10.

4. Stitt M, Hurry V. A plant for all seasons : alterations in photosynthetic carbon metabolism during cold acclimation in Arabidopsis. Curr Opin Plant Biol. 2002:5:199-206.

5. Uematsu K, Suzuki N, Iwamae T, Inui M, Yukawa H. Increased fructose 1,6bisphosphate aldolase in plastids enhances growth and photosynthesis of tobacco plants. J Exp Bot. 2012;63:3001-9.
6. Rutter WJ. Evolution of aldolase. Fed Proc. 1964;23:1248-57.

7. Ronimus RS, Morgan HW. Distribution and phylogenies of enzymes of the Embden-Meyerhof-Parnas pathway from archaea and hyperthermophilic bacteria support a gluconeogenic origin of metabolism. Archaea. 2003;1: 199-221.

8. Flechner A, Gross W, Martin W, Schnarrenberger C. Chloroplast class I and class II aldolases are bifunctional for fructose-1,6-biphosphate and sedoheptulose-1,7-biphosphate cleavage in the Calvin cycle. FEBS Lett. 1999;447:200-2.

9. Patron NJ, Rogers MB, Keeling PJ. Gene replacement of Fructose-1,6Bisphosphate Aldolase supports the hypothesis of a single photosynthetic ancestor of Chromalveolates. Eukaryot Cell. 2004;3:1169-75.

10. Haake V, Geiger M, Walch-Liu P, C Engels R, Zrenner M. Stitt, changes in aldolase activity in wild-type potato plants are important for acclimation to growth irradiance and carbon dioxide concentration, because plastid aldolase exerts control over the ambient rate of photosynthesis across a range of growth conditions. Plant J. 1999;17:479-89.

11. Haake V, Zrenner R, Sonnewald U, Stitt M. A moderate decrease of plastid aldolase activity inhibits photosynthesis, alters the levels of sugars and starch, and inhibits growth of potato plants. Plant J. 1998;14:147-57.

12. Poolman MG, Fell DA, Thomas S. Modelling photosynthesis and its control. J Exp Bot. 2000;51:319-28

13. Cai $B, L i ~ Q, X u Y$, Yang $L, B i H, A i X$. Genome-wide analysis of the fructose 1,6-bisphosphate aldolase (FBA) gene family and functional characterization of FBA7 in tomato. Plant Physiol Biochem. 2016;108:251-65.

14. Purev M, Kim MK, Samdan N, Yang D. Isolation of a novel fructose-1,6bisphosphate aldolase gene from Codonopsis lanceolata and analysis of the response of this gene to abiotic stresses. Mol Biol. 2008;42:179-86.

15. Lu W, Tang X, Huo Y, Xu R, Qi S, Huang J, Zheng C, Wu C. Identification and characterization of fructose 1,6-bisphosphate aldolase genes in Arabidopsis reveal a gene family with diverse responses to abiotic stresses. Gene. 2012; 503:65-74.

16. Qiu H, Zhang L, Liu C, He L, Wang A, Liu H, Zhu J. Cloning and characterization of a novel dehydrin gene, SiDhn2, from Saussurea involucrata Kar. Et Kir. Plant Mol Biol. 2014;84:707-18.

17. Guo X, Zhang L, Zhu J, Liu H, Wang A. Cloning and characterization of $\mathrm{SiDHN}$, a novel dehydrin gene from Saussurea involucrata Kar. Et Kir. That enhances cold and drought tolerance in tobacco. Plant Sci. 2017;256:160-9.

18. Li J, Liu H, Xia W, Mu J, Feng Y, Liu R, Yan P, Wang A, Lin Z, Guo Y. De novo Transcriptome sequencing and the hypothetical cold response mode of Saussurea involucrata in extreme cold environments. Int J Mol Sci. 2017;18:1155

19. M J Vahdati K, Dandekar A, Leslie C, Uratsu S, Hackett W, Negri P, McGranahan G. Rooting and other characteristics of a transgenic walnut hybrid (Juglans hindsii $x$ J. regia) rootstock expressing rolABC. J Am Soc Horticultural Sci. 2002;127:724-8.

20. Khodadadi F, Tohidfar M, Vahdati K, Dandekar AM, Leslie CA. Functional analysis of walnut polyphenol oxidase gene (JrPPO1) in transgenic tobacco plants and PPO induction in response to walnut bacterial blight. Plant Pathol. 2020;69:756-64

21. Bolagh FRQ, Solouki A, Tohidfar M, Mehrjerdi MZ, Darbandi Al, Vahdati K Agrobacterium-mediated transformation of Persian walnut using BADH gene for salt and drought tolerance. J Horticultural Sci Biotechnol. 2020;1812446:1-10.

22. Goharrizi MASB, Dejahang A, Tohidfar M, Darbandi Al, Carillo N, Hajirezaei MR, Vahdati K. Agrobacterium mediated transformation of somatic embryos of Persian walnut using fld gene for osmotic stress tolerance. J. Agr. Sci. Tech. 2016;18:423-35.

23. Habibie A, Yazdani N, Saba MK, Vahdati K. Ascorbic acid incorporated with walnut green husk extract for preserving the postharvest quality of cold storage fresh walnut kernels. Sci Hortic. 2019:245:193-9.

24. VK Aslani Aslamarz A, M Rahemi D. Hasani, Cold-Hardiness Evaluation of Persian Walnut by Thermal Analysis and Freezing Technique. Acta Horticulturae. 2009:861:269-72.

25. V.K, Aslani Aslamarz A, Hasani D, Rahemi M, Leslie CA. Cold Hardiness and its Relationship with Proline Content in Persian Walnut. Eur J Horticultural Sci. 2011;76: 84-90.

26. V.K, Aslani Aslamarz A, Hasani D, Rahemi M, Leslie CA. Supercooling and Cold-hardiness of Acclimated and Deacclimated Buds and Stems of Persian Walnut Cultivars and Selections. HortScience. 2010:45:1-6.

27. Mohapatra S, Mittra B. Protein glutathionylation protects wheat (Triticum aestivum Var. Sonalika) against Fusarium induced oxidative stress. Plant Physiol Biochem. 2016;109:319-25. 
28. Zhang G, Liu Y, Ni Y, Meng Z, Lu T, Li T. Exogenous calcium alleviates low night temperature stress on the photosynthetic apparatus of tomato leaves. Plos One. 2014;9.

29. Xie Q, Shen K, Hao X, Nam PN, Bt NH, Chen CH, Zhu C, Lin YC, Hsiao C. The complete chloroplast genome of Tianshan snow Lotus (Saussurea involucrata), a famous traditional Chinese medicinal plant of the family Asteraceae. Mitochondrial DNA. 2017;28:1-2.

30. Kossmann J, Sonnewald U, Willmitzer L. Reduction of the Chloroplastic Fructose-1,6-Bisphosphatase in transgenic potato plants impairs photosynthesis and plant-growth. Plant J. 1994;6:637-50.

31. Du H, Wu N, Fu J, Wang S, Li X, Xiao J, Xiong L. A GH3 family member, OsGH3-2, modulates auxin and abscisic acid levels and differentially affects drought and cold tolerance in rice. J Exp Bot. 2012;63:6467-80.

32. Gill SS, Tuteja N. Reactive oxygen species and antioxidant machinery in abiotic stress tolerance in crop plants. Plant Physiol Biochem. 2010:48:909-30.

33. Murata N, Takahashi S, Nishiyama Y, Allakhverdiev SI. Photoinhibition of photosystem II under environmental stress. Biochim Biophys Acta. 2007; 1767:414-21.

34. Baker NR, Rosenqvist E. Applications of chlorophyll fluorescence can improve crop production strategies: an examination of future possibilities. J Exp Bot. 2004;55:1607-21.

35. Ding F, Wang M, Zhang S, Ai X. Changes in SBPase activity influence photosynthetic capacity, growth, and tolerance to chilling stress in transgenic tomato plants. Sci Rep. 2016;6:32741.

36. M.F.Y. HONG MA, AND Elliot M. MEYEROWITZ, Molecular cloning and characterization of GPAl, a G protein a subunit gene from Arabidopsis thaliana, Proc. Natl. Acad. Sci, 87, 1990 3821-3825,

37. Dan Y, Yan H, Munyikwa TRI, Dong J, Zhang Y, Armstrong CL. MicroTom-a high-throughput model transformation system for functional genomics. Plant Cell Rep. 2006;25:432-41.

38. Quan R, Shang M, Zhang H, Zhao Y, Zhang J. Improved chilling tolerance by transformation with betA gene for the enhancement of glycinebetaine synthesis in maize. Plant Sci. 2004;166:141-9.

39. Giannopolitis CN, Ries SK. Superoxide dismutases: I. occurrence in higher plants. Plant Physiol. 1977;59:309-14.

40. Polle A, Otter T, Seifert F. Apoplastic peroxidases and lignification in needles of Norway spruce (Picea abies L.). Plant Physiol. 1994;106:53-60.

41. Cakmak I, Strbac D, Marschner H. Activities of hydrogen peroxide-scavenging enzymes in germinating wheat seeds. J Exp Bot. 1993;41:127-32.

42. Miyagawa Y, Tamoi M, Shigeoka S. Evaluation of the defense system in chloroplasts to Photooxidative stress caused by Paraquat using transgenic tobacco plants expressing catalase from Escherichia coli. Plant Cell Physiol. 2000:41:311-20.

\section{Publisher's Note}

Springer Nature remains neutral with regard to jurisdictional claims in published maps and institutional affiliations.

Ready to submit your research? Choose BMC and benefit from:

- fast, convenient online submission

- thorough peer review by experienced researchers in your field

- rapid publication on acceptance

- support for research data, including large and complex data types

- gold Open Access which fosters wider collaboration and increased citations

- maximum visibility for your research: over $100 \mathrm{M}$ website views per year

At $\mathrm{BMC}$, research is always in progress.

Learn more biomedcentral.com/submissions 